\title{
Research on the Natural Image Classification and Segmentation Algorithm based on GPU and Neural Network
}

\author{
Liwei Chen ${ }^{1}$ \\ ${ }^{1}$ College of Computer Science and Technology, SouthWest University of Science and Technology, \\ Mianyang,621010,China
}

\begin{abstract}
In this paper, we conduct research on the natural image classification and segmentation algorithm based on GPU and neural network. The application of image segmentation is very broad, almost appeared in all areas related to image processing, and involved in various types. With the fast development of computing technology and integrated circuit technology, the renewal speed of graphics hardware. Our method combines the GPU with network to optimize the traditional image segmentation and classification methods which will be meaningful. In the future, we will focus our attention on the hardware deployment of the GPU to modify the current approach.
\end{abstract}

Keywords: Image Classification; Image Segmentation; GPU and Neural Network.

\section{Introduction}

Image segmentation and classification is the fundamental challenging problem in machine vision, and image processing, image analysis, pattern recognition and tracking of the important content. In the field of multiple target segmentation, the goal of image segmentation based on image with similar properties or a single property, such as grey value distribution, edge profile, structure, color and shape, such as image segmentation and extracts the interested parts for multiple areas. The purpose of image segmentation is to divide the image into several mutually disjoint regions, each region has a consistency, and attribute feature is an obvious difference between adjacent areas. The application of image segmentation is very broad, almost appeared in all areas related to image processing, and involved in various types. Based on region segmentation method is based on direct search for regional segmentation technology, specific algorithm with regional growth and regional separation and merger algorithm. Based on region extraction method has two basic forms: one is a regional growth, from a single pixel, gradually merging to form the required segmented regions and another from global, the cutting step by step to the segmented regions. In actual use of usually is a combination of these two basic forms. The algorithms for some complex object definition of complex scene segmentation or segmentation of some of the natural scenery and similar lack of prior knowledge of image segmentation, the effect is ideal. Segmentation method based on edge detection is attempted to solve the problem by detecting the edge of the different areas, usually on the edge of gray value between different regions tend to be changed, this is the main assumptions of the edge detection method are realized. Edge detection can get grey value of the local intensity change, and regional segmentation is capable of detecting characteristics of similarity and uniformity [1-3].

Accompanied by the rise of grade PC microcomputer and popularization, the years have taken place in most of the application of computer graphics from the workstation to the big transfer with microcomputer, and the transfer occurs even in real-time applications such as virtual reality, computer simulation. The real-time application of the popularity of computer games is also a sign of this development. All this happen to a large extent, is derived from the development and innovation of 
graphics hardware. With the development of computing technology and integrated circuit technology, the renewal speed of graphics hardware. So when the function of the GPU is more and more strong, related to graphic processing and naturally from CPU to GPU.

An important feature of neural network is could be obtained by learning from environmental knowledge and improve their own performance. To improve the performance of the network, usually in a certain predetermined measure, through to the network setting and adjustment of each neuron initial parameter values in the step by step. The ultimate measure of learning is to make the error between the actual output and the output should be the minimum. Artificial neural network knowledge storage capacity is very large. In neural network, the storage of knowledge and information is distributed physical contact between neurons. It said scattered and stored in the whole of the neurons in the network and its attachment. Each neuron and their attachment part of said information only, and not a complete specific concept. Only through comprehensive effect of various neurons distributed can express specific concepts and knowledge. Because there is so much the number of neurons in artificial neural network and the network storage large information capacity which makes it has strong ability of uncertainty information processing.

In this paper, we conduct research on the natural image classification and segmentation algorithm based on GPU and neural network. Artificial neural network with the current computer is different, is a kind of nonlinear processing units. Only when the synthesis processing results of the input signal of neurons after more, a certain threshold method should to output a signal. It breaks through the general traditional on the basis of the linear processing of the digital electronic computer. In the figure we show the combination of natural image segmentation and classification with neural network.
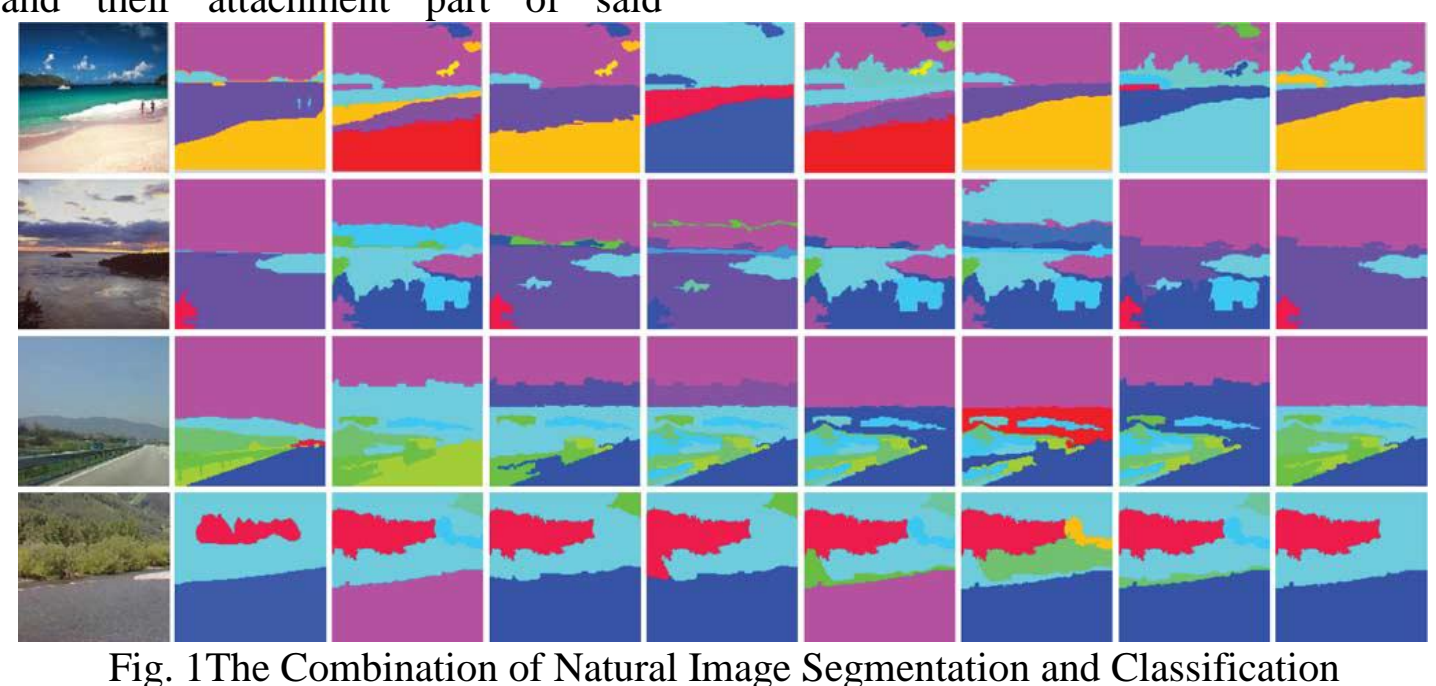

Fig. 1The Combination of Natural Image Segmentation and Classification

\section{Our Proposed Algorithm and Methodology}

The Image Classification. Image classification is an important research direction in the field of computer vision, has been widely applied in real life. In the image of there is the semantic gap between low-level features and advanced concepts. The wider the image field Interval. So this kind of system has made some success, but the retrieval results are often disappointing. Another kind of method is based on the explanation of the method. After the analysis of characteristics of image and extract the contents of but direct semantic explanation, allowing users to conceptualize the query. We believe that the conceptualization of the query is more 
practical significance. Therefore, limit the field of image, to narrow the semantic gap between low-level features and high-level concepts, and with the help of a machine learning method of automatic image class model used for image retrieval, could be a meaningful way. Our approach is to use support vector machine as a learning machine, study the content of the image in the field of natural images, learn to image classification model is used for image retrieval. The formula one defines the objective function.

$$
\min _{u, v} \sum_{i=1}^{N}\left\|y_{i}-U v_{i}\right\|^{2} \quad \text { s.t. } \quad \operatorname{Card}\left(v_{i}\right)=1
$$

In the image of the class model is set up, we avoid the need for explicit prior knowledge, learning to model is implicit in the learning machine.

$$
\min _{u, v} \sum_{i=1}^{N}\left\|y_{i}-U v_{i}\right\|^{2}+\lambda\left\|v_{i}\right\|_{1} \quad \text { s.t. } \quad \operatorname{Card}\left(v_{i}\right)=1
$$

Every image in the class contains from belongs to the class at the same time contains information has nothing to do with the class the content is protean. Good features should be able to eliminate the noise to seize the characteristics of the image class nature. Invariance can be used to depict this nature. Color histogram of color appear simply frequency statistics not consider color spatial distribution. It is thus distinguish ability is very weak cannot distinguish between colors are densely distributed and the distribution of the loose situation. In line with color histogram and color vector is different color not only from the perspective of the frequencies of color to describe the color distribution information of images and depict the relationship between the spatial distributions of all sorts of color in the image. It is through the statistics of the image changes in two kinds of color in various distances.

The Principles of Neural Network. Artificial neural network is the understanding of the organization structure and operation mechanism in the brain, abstract, simplification and simulation of an information processing system. ANN based on a large number of simple processing units for interconnection, form a complex network system. The processing unit and the connection mode are borrowed from the neurons interconnection structure and the network connection mechanism, the connections between neurons are not simply in the transmitting signal channel, they are a weighted coefficient, can the synaptic strength of biological neurons in the nervous system to imitate. The topology of the nervous system stability rules and limits the nature of the neural network and the size of the information processing ability. According to the different methods of interconnection, the neural network can be divided into type feedforward network and the feedback network. Feedforward network there is no feedback loop between neurons, and the feedback network because of the feedback loop, therefore has the short-term memory function. From the point of effect and the feedforward network is primarily a function mapping, can be used for pattern recognition and function approximation. The following formula shows the process.

$$
y=f\left[\sum_{i=1}^{m} x_{i} w_{i}-\theta\right]
$$

Artificial neural network is composed of a large number of neurons widely interconnected system and the structure characteristics of it decides the artificial neural network has the ability of high speed information processing. Because of the structure characteristics of artificial neural network and the distributed characteristics of the information is stored, relative to that of other identification system, such as expert system has another remarkable advantages: robustness. Biological neural network won't because of the loss of individual neurons lose memory of the original model artificial neural network has a similar situation. For some reason, network hardware implementation and software implementation of 
a failure or a certain type of neuron, the entire network can still continue to work.

The GPU based Image Processing Method. General calculation based on the GPU is developed with the development of graphics chips, while previous graphics card is mainly aimed at application of graphics itself, such as lighting calculation, depth detection, rasterization, anti-aliasing, and so on, but for other more general applications have appeared, such as the use of color objects to mark the serial number, etc. CPU is essentially a scalar calculation model, and fewer cell, mainly for complex control delay rather than a high bandwidth and low the number of optimization. Before the programmability popularization, normally these methods are combined to use, in order to meet the different specific issues, several methods but the biggest problem is not enough precision and flexibility, because they belong to the method of the fixed rendering pipeline, the precision of each color channel only eight, so have to advance to the data in the process of calculation rules, and to ensure that the rules of intermediate results.

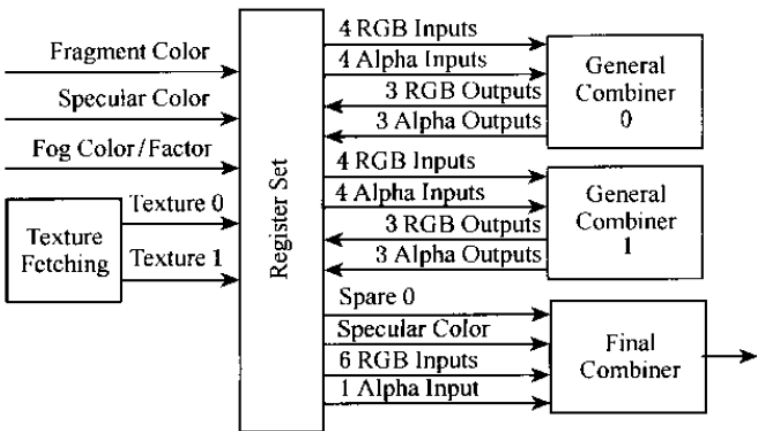

Fig. 2The Organization and Structure of GPU

Although the vertex programming has support branch operation and circulation, but cannot access the texture data, and graphics hardware pixel processing power is an order of magnitude higher than the vertex processing capabilities and pixel filling rate is far higher than triangular transmission speed, and the current pixel rendering pipeline than vertex rendering pipeline, so the pixel program is more suitable for general computing.

The Combined Method and the Numerical Analysis. Natural image classification method based on the layout. It is qualitative spatial relations between regions of the image as a characteristic, thus, within the scope of natural images, can capture the concept of image information. In the following figure three, we illustrate the numerical simulation result for the proposed approach.
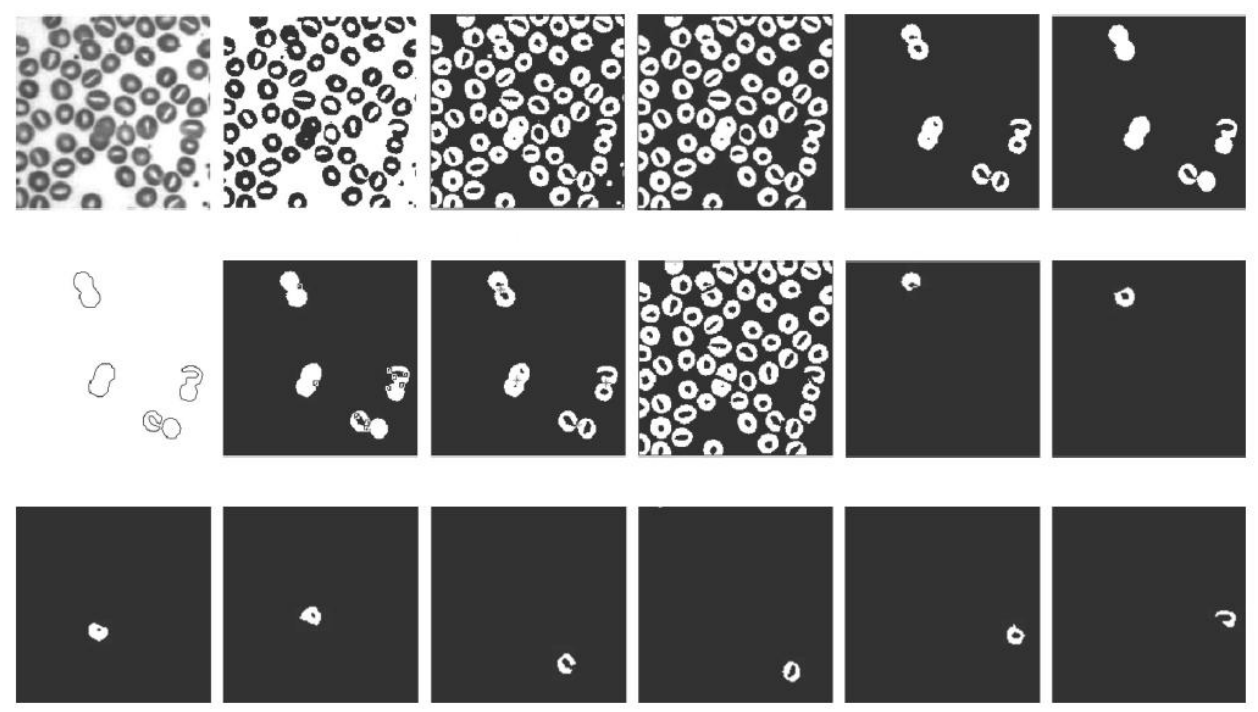

Fig. 3The Experimental Result of the Proposed Methodology 


\section{CONCLUSIONS}

In this paper, we conduct research on the natural image classification and segmentation algorithm based on GPU and neural network. Close value method is a simple and effective method of image segmentation, it uses one or several standards the grayscale of the image is divided into several parts, that belong to the same part of the pixel is the same object. For the target and the background of image grayscale have obvious difference, the gray histogram distribution is bimodal the two cross peak respectively and the corresponding to the target and the background in the image, the valleys correspond to image edge. Our method combines the advances of GPU and neural network to enhance the general performance of the segmentation and classification steps.

\section{Acknowledgement}

This research is financially supported by the artificial intelligence laboratory in Sichuan province, the key laboratory open fund (NO. 2014RYY03).

\section{References}

[1] Douillard B, Underwood J, Vlaskine V, et al. A Pipeline for the Segmentation and Classification of 3D Point Clouds[M]// Experimental Robotics. Springer Berlin Heidelberg, 2014:585-600.

[2] Chen, Y. F., Huang, P. C., Lin, K. C., Lin, H. H., Wang, L. E., \& Cheng, C. C., et al. (2014). Semi-automatic segmentation and classification of pap smear cells.. Biomedical \& Health Informatics IEEE Journal of, 18(1), $94-108$.

[3] Mettes P, Tan R T, Veltkamp R. On the Segmentation and Classification of Water in Videos[J]. International Conference on Computer Vision Theory \& Applications, 2014. 